\title{
Successful Jugular Implantable Defibrillator Lead extraction with Bidirectional Rotational Mechanical Sheath
}

\author{
Federico Migliore $^{1}$, Pasquale Valerio Falzone ${ }^{2}$, Pietro Bernardo Dall'Aglio ${ }^{2}$, Michele \\ Piazza $^{2}$, Vincenzo Tarzia ${ }^{2}$, and Gino Gerosa ${ }^{3}$ \\ ${ }^{1}$ University of Pafova \\ ${ }^{2}$ University of Padova School of Medicine and Surgery \\ ${ }^{3}$ University of Padua Department of Cardiac Thoracic and Vascular Science
}

November 12, 2020

\begin{abstract}
Evolution RL sheath with its bidirectional rotational mechanism is an effective and safe technique for transvenous lead extraction of chronically implanted leads. However, data about powered mechanical sheath through jugular vein are lacking. Our report suggest that bidirectional rotational mechanical sheath may represent an effective and safe approach for removal of chronically implanted jugular lead.
\end{abstract}

\section{Successful Jugular Implantable Defibrillator Lead extraction with Bidirectional Rotational Mechanical Sheath}

Federico Migliore, MD, PhD, Pasquale Valerio Falzone, MD, Pietro Dall'Aglio MD, Michele Piazza MD, Vincenzo Tarzia, MD, PhD, Gino Gerosa MD,

Department of Cardiac, Thoracic, Vascular Sciences and Public Health, University of Padova, Padova, Italy

Short title: Jugular ICD lead extraction

Funding statement: None

Author disclosures statement: None

Correspondence to: Prof. Federico Migliore MD, PhD, FESC, FEHRA

Department of Cardiac, Thoracic, Vascular Sciences and Public Health, University of Padova, Padova, Italy

Via N. Giustiniani 2, 35121 Padova, Italy; Tel.+39 0498212332

e-mail:federico.migliore@libero.it

A 51-year-old female with hypertrophic cardiomyopathy underwent single-chamber implantable cardioverterdefibrillator (ICD) implantation in 2010. Because of lead failure (single coil, active fixation) transvenous lead extraction (TLE) was scheduled. Although the description of the previous lead implantation reported an access through the left subclavian vein, the preoperative chest x-ray showed a possible extravascular course of the lead above the left clavicle (Figure 1A). Cardiac computed tomography demonstrated a course of the lead anteriorly to the left clavicle with a subcutaneous course and through the left internal jugular vein (Figure 1B). The procedure was performed under general anesthesia in a hybrid operating room. A stiff guidewire from the right femoral vein to the right internal jugular vein for potential use of the bridge occlusion balloon in case of vascular lacerations. A skin incision under the clavicle and a second one at the lead insertion site in 
the neck over the internal jugular vein were made. The subcutaneous portion of the lead was separated from the scar tissue by blunt dissection over the bone and retrieved from the tunnel through the incision in the neck. Manual traction was attempted unsuccessfully with a locking stylet. The lead was successful extracted with powered-mechanical sheath and outer sheath using a step wise approach (1) (first using Evolution Shortie RL 11F and than Evolution RL 11F, Cook Medical, Bloomington, IN, USA) through the jugular vein (Figure 1C-F) Multiple adhesions found in entry vein site, in the brachiocephalic vein and superior vena cava. No complications were encountered during and after the procedure. A new lead was implanted through the left axillary vein. Evolution RL sheath with its bidirectional rotational mechanism is an effective and safe technique for TLE of chronically implanted leads $(1,2)$. However, data about powered mechanical sheath through jugular vein are lacking. Our report highlights first he importance of preprocedural imaging before TLE and suggest that bidirectional rotational mechanical sheath could represent an effective and safe approach for removal of chronically implanted jugular leads.

\section{Figure legend}

Preoperative chest x-ray (A ) and cardiac computed tomography (B ) demonstrated a course of the lead anteriorly to the left clavicle with a subcutaneous course and through the left internal jugular vein. Evolution Shortie RL 11F sheath (C andD ) and Evolution RL 11F (E ) sheath through the jugular vein were used for removed the lead. The extracted lead $(\mathbf{F})$. LIJV=left internal jugular vein.

\section{References}

1.Migliore F, Testolina M, Sagone A et al. Multicenter experience with the Evolution RL mechanical sheath for lead extraction using a stepwise approach: Safety, effectiveness, and outcome. Pacing Clin Electrophysiol. 2019;42:989-997

2.Sharma S, Ekeruo IA, Nand NP et al. Safety and Efficacy of Transvenous Lead Extraction Utilizing the Evolution Mechanical Lead Extraction System: A Single-Center Experience. JACC Clin Electrophysiol. 2018;4:212-220

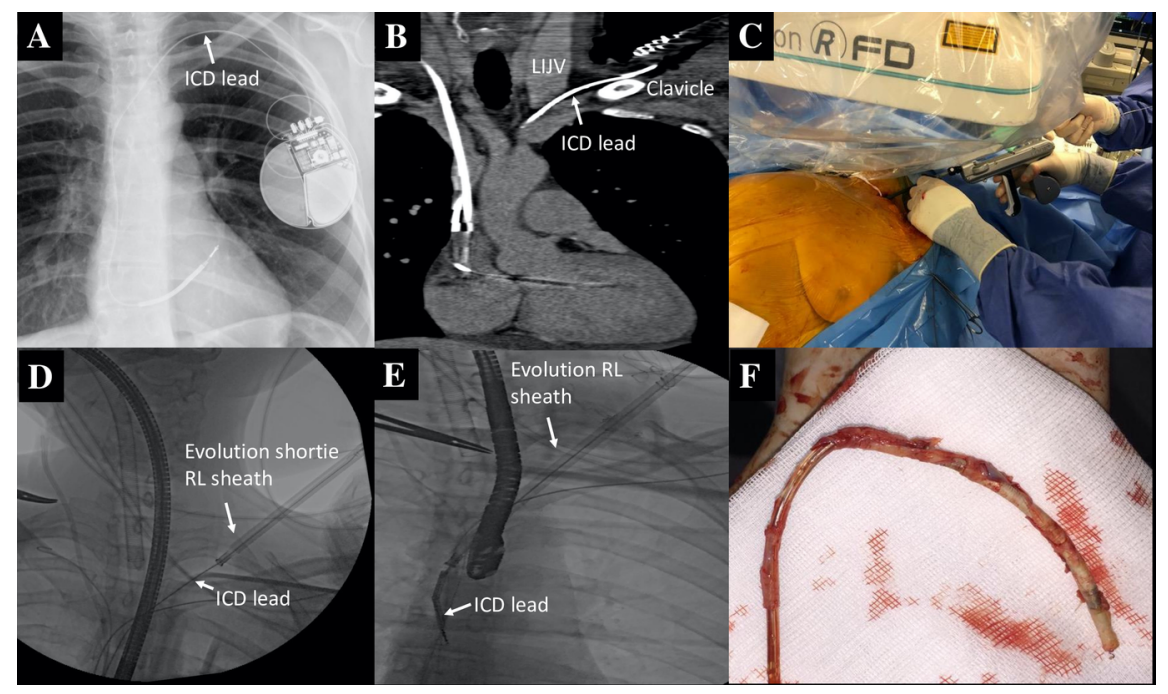

